Ute Daub

\title{
Der Frankfurter Euthanasie-Prozeß
}

Seit Anfang des Jahres erhält die Häßlichkeit des Raums A I 46 im alten Frankfurter Gerichtsgebäude wöchentlich für zwei Stunden einen historischen Sinn. Die Zeit scheint in diesem Saal geschmolzen zu sein und in der Schmelze ist - bis auf jene "1000 Jahre - alles konserviert: Zugemauerte wilhelminische Fenster, braun gestrichene Holzbänke aus der Zwischenkriegszeit, hohe Polsterstühle für das hohe Gericht aus der Ära der neuen Sachlichkeit, später mit rotem Kunstleder und antiquisierten Polsternägeln wieder aufgefrischt, dahinter an der Stirnseite ein monumentales Wappen aus der Nierentischära, schließlich die für die Ewigkeit stabil verankerte Glaswand aus der Zeit der RAF-Prozesse. Blickt man sich um, sieht man die Zuschauer wie in einem Schaufenster. Alle in der Haltung von schwerhörigen Greisen: weit vornüber gebeugt, auf der äußersten Stuhlkante sitzend, halboffene Münder, Stirnfalten, die Hände hinter den Ohrmuscheln. Hier ist seit Ende Januar ein NS-Verbrechensprozeß, der staatlichen Massenmord an Klienten der Psychiatrie verhandelt, zu Hause.

\section{Vorgeschichte}

Es ist wahrscheinlich der letzte, da im Sommer, aus nach wie vor nicht nachvollziehbaren rechtlichen Gründen, klammheimlich das drei Jahre währende Ermittlungsverfahren gegen den in die "Euthanasie-Aktion « verstrickten forensischen Gutachter Hans-Joachim Rauch von Oberstaatsanwalt Schmidt, Heidelberg, eingestellt wurde.

Bei Fritz Bauers Ermittlungen gegen den "Euthanasie «-Gutachter Heyde, alias Dr. Sawade, Anfang der sechziger Jahre, gelang es, hinter den Tarnnamen "Dr. Schmitt" den Stuttgarter Frauenarzt Aquilin Ullrich sowie als "Dr. Rieper" bzw. „Dr. Keller" seinen Kollegen Heinrich Bunke aus Celle zu reidentifizieren. Gemeinsam mit Klaus Endruweit, Hildesheim (alias "Dr. Bader«), und Nikolaus Borm (»Dr.Storm«) aus Uetersen warf ihnen der Generalstaatsanwalt 1965 vor, »heimtückisch, grausam, aus niedrigen Beweggründen, vorsätzlich und mit Überlegung jeweils mehrere tausend Menschen getötet zu haben «. ${ }^{1}$ Bei Prozeßbeginn 1966 wird das Verfahren gegen Borm abgetrennt, denn man glaubt, ihm noch weitere Straftaten nachweisen zu können. ${ }^{2} 1967$ wird das Urteil gesprochen: Die Tatbestandsmerkmale des $\$ 2$ I I StGB seien erfüllt; die Tötung sei heimtückisch erfolgt, Rechtfertigungsgründe gebe es nicht. Grausam sei die Tötung allerdings nicht gewesen, sei doch »die Tötung durch Kohlenmonoxyd eine der humansten Tötungsarten. Da die Tötung in den Gaskammern ... durch chemisch reines Kohlenmonoxyd erfolgte, sind den Opfern keinerlei körperliche Schmerzen oder Qualen zugefügt worden ... Da chemisch reines Kohlenmonoxyd farb- und geruchlos ist, ergaben sich auch bis zum Eintritt der Bewußtlosigkeit keinerlei unangenehme oder gar schmerzhafte Empfindungen. Die Tötung durch Kohlenmonoxyd verursachte

\footnotetext{
1 Zitıer nach Ernst Klee, Die Strafsache gegen Ullrıch, Bunke, Endruwe1t, Frankfurt/M 198 s, S. 23. Ks I/ 66.

2 a. a. O., S. 27
} 
auch keinerlei seelische Qualen bei den Opfern ... die Bewußtlosigkeit (ist) bei allen Kranken nahezu gleichzeitig eingetreten ... (deswegen) hatte der widerstandsfähigere Teil der Kranken nicht etwa dadurch seelische Qualen zu erleiden, daß er bei dem anderen Teil die Gifteinwirkung bemerkte und deshalb selbst von Todesangst befallen wurde ${ }^{3}{ }^{3}$

Die Angeklagten wurden freigesprochen, denn sie hätten in einem unvermeidbaren Verbotsirrtum gehandelt, deswegen keine Schuld auf sich geladen. Sie seien davon ausgegangen, »daß sie nur bei der Tötung von Geisteskranken ohne natürlichen Lebenswillen mitwirkten und daß deren Tötung erlaubt war ... Es lag nahe, daß sie sich sagen mußten, daß die ... angewandten Täuschungen bei Irren der untersten Kategorie überflüssig wären. Die Angeklagten hatten jedoch nur unzureichende theoretische und keinerlei praktische Kenntnisse auf dem Gebiet der Psychiatrie und Neurologie.« Der BGH hob im Sommer 1970 das Urteil auf: Auf allerhöchstens ein Prozent der Getöteten habe die Bezeichnung "niedergeführte Existenzen « zugetroffen. Kundgebungen des Lebenswillens könne jeder wahrnehmen, »ohne $\mathrm{da} ß$ es dazu vertiefter psychiatrischer oder überhaupt medizinischer Fachkenntnisse bedurfte $\ll^{4}$.

Das Verfahren gegen Ullrich, Bunke und Endruweit wurde aus prozeßökonomischen Gründen wieder mit dem Verfahren gegen Borm verbunden; 1972 wurde erneut verhandelt, allerdings nur noch gegen Borm, denn das Verfahren gegen Ullrich, Bunke und Endruweit wurde wegen deren Verhandlungsunfähigkeit vorläufig eingestellt. Borm wurde erneut freigesprochen: Er habe geglaubt, es habe sich bei den Geisteskranken »ausnahmslos um völlig verblödete, abgebaute Existenzen gehandelt, für die der Tod ... eine Erlösung bedeutet habe«, ihm habe »unwiderlegbar das Bewußtsein der Rechtswidrigkeit « seines Tuns gefehlt: »In den entscheidenden Jahres seines Heranwachsens ... hat er kaum etwas anderes vernommen als die Verherrlichung nationalsozialistischen Gedankengutes. Er ist aufgewachsen in einem Beamtenhaushalt mit der dort ... vorhandenen staatstreuen Gesinnung und dem unbedingten Glauben an die Gesetzmäßigkeit hoheitlichen Gebarens. «s

Dieses Urteil hielt 1974 der Überprüfung durch den BGH stand. Dem 76 jährigen ehemaligen Mitglied der Leibstandarte Adolf Hitler und der SS-Totenkopfverbände erlauben der schleswig-holsteinische Sozialminister, die Kassenärztliche Vereinigung und die Landesärztekammer immer noch als niedergelassener Arzt für Allgemeinmedizin zu praktizieren. Ärztliche Gutachter und das Frankfurter Landgericht gestatteten Ullrich, Bunke und Endruweit, fünfzehn Jahre lang zu praktizieren, zu operieren und verhandlungsunfähig zu sein. Versuche der Regierungspräsidenten, ihnen vorläufig die Approbation zu entziehen, scheiterten an Solidaritätsbekundungen Tausender, von Bundes- und Landtagsabgeordneten, von Ministern, Vereinen, kirchlichen Organisationen, Bürgermeistern, zuletzt erfolgreichen Anfechtungsklagen. ${ }^{6}$

\footnotetext{
a. a. O., S. $30 \mathrm{ff}$

42 StR $353 / 68$

$5 \mathrm{Ks} \mathrm{I} / 66$.

6 Endruweıt scheute sıch nıcht, den Brıef eınes Bürgermeısters an den Regırungspräsıdenten weıterzuleıten, in dem es heißt: „Bereits jetzt werden in nicht zu übersehendem Maße peınliche und dem Ansehen der Regıerung sehr schädliche Vergleıche mıt Willkürmaßnahmen des Dritten Reıches gezogen ... (wir, die wir) durch das schwere Schıcksal der Vertreibung so viel Unrecht selbst miterleben, mit ansehen mußten, wı wehrlose Frauen, Kinder, Greıse und Männer willkürlich und ohne jeden Grund erschossen oder oft auf bestıalische Weise umgebracht wurden, (uns) ist es enfach unbegreiflich, wie man einem so hochgeschätzten, angesehenen und ehrbaren Menschen wie Dr. Endruweit es ist, einfach durch Zwangsmaßnahmen entehrt."A.a.O., S. $26 \mathrm{f}$.
} 
Erst 1985 gelang es der 22. Strafkammer des Landgerichts, die Angeklagten mit Hilfe weiterer ärztlicher Gutachten für eingeschränkt verhandlungsfähig zu erklären. Auch das von den Angeklagten angerufene Bundesverfassungsgericht wollte an dieser Entscheidung nichts ändern. Das Verfahren gegen Endruweit mußte gleichwohl vorläufig eingestellt werden: Zwei Ärzte bescheinigten ihm, einen Nierenstein zu haben, der Koliken verursachen könne, und attestierten ihm - der Kompetenz des Gerichts vorgreifend - Verhandlungsunfähigkeit. Endruweit verzichtet auf eine Behandlung seines Leidens und das Gericht unterläßt es bislang, $\$ 23$ ra StPO anzuwenden und ihn als einen deutschen Terroristen anzusehen, gegen den in Abwesenheit verhandelt werden kann, hat er seine Verhandlungsunfähigkeit selbst herbeigeführt.

\section{Die "T4-Aktron"}

Bunke und Ullrich »arbeiteten « als Angestellte der »Kanzlei des Führers $(\mathrm{KdF})$ in den Tötungsanstalten Brandenburg/Havel und Bernburg/Saale. »Um das zu tun, mußten die Nazis schweigen. Hätten sie ihre Taten in Worte fassen können, hätten sie das nicht tun können «, meint Claude Lanzmann. ${ }^{7}$ In dem Sinn »schweigen « wir noch immer, haben wir doch für dieses Arbeitsprodukt der KdF keinen anderen Namen als " $\mathrm{T}_{4}$-Aktion $4 .{ }^{8}$ Darunter wird der zweite Teil der "Euthanasie-Aktion « zwischen August 1939 und August I94 I verstanden. Diese "Aktion « ist der erste Massenmord unter Verwendung von Gas an Erwachsenen, nachdem die Kinder"Euthanasie« bereits begonnen hatte. Die " $\mathrm{T}_{4}$-Aktion « ist das einzige Massenverbrechen des Nationalsozialismus, das durch - am Ende - öffentliche Proteste nachhaltig behindert wurde. Entgegen einem weit verbreiteten Vorurteil wurden auch nach der Predigt des Münsterraner Bischofs von Galen Bewohner von Heimen und Krankenhäusern weiter systematisch umgebracht, in einigen Anstalten noch Monate nach der Kapitulation. Im Unterschied zu $\mathrm{T}_{4}$, bei der 70273 Menschen ermordet wurden, ist die Zahl der Opfer dieser sogenannten »wilden Euthanasie nicht bekannt; man weiß nur, daß es insgesamt mehr als 100000 Menschen gewesen sein müssen.

Die " $\mathrm{T}_{4}$-Aktion « wurde sorgfältig geplant und implementiert: Diverse Tarnorganisationen wurden gegründet, Expertenkommissionen gebildet, Tötungsmethoden wissenschaftlich evaluiert, Meldebögen pretests unterzogen, Nomenklaturen wurden eingeführt, Sonderstandesämter eingerichtet, "Absteckabteilungen « wurden gegründet, die die faktische Korrelation von Herkunftsort und Tötungsanstalt dadurch zu verschleiern hatten, daß die "Trostbriefe " den Poststempel seltener genannter Tötungsanstalten erhielten. Zwischenlager wurden »erfunden ", selbst Instrumente zur Kontrolle und Zensur privater Todesanzeigen, die gelegentlich eindeutig mehrdeutig formuliert waren, wurden geschaffen. ${ }^{9}$ Mobile Selektionskomitees (die sogenannten Ärztekommissionen) wurden gebildet, um den vereinzelt auftretenden passiven Widerstand einiger Institutionen zu brechen.

Selektionskriterien wurden abgestimmt und hinsichtlich ihrer Trennschärfe und politischen Opportunität "praktisch geprüft.. Schließlich wurde unter der Über-

\footnotetext{
7 Im Interview zu seınem Film "Shoah".

8 In der Tiergartenstraße 4 in Berlin, in der warısıerten « ehemaligen Villa von Max Liebermann - auf ihrem Grundstück steht heute die Philharmone - befand sıch eıne Zeitlang der Sitz dieser Organısation.
}

9 Kurt Nowak, «Euthanasıe" und Sterilisıerung im "Drıtten Reıch«, Göttıngen 1978. 
schrift "Was ist bisher in den einzelnen Anstalten ... desinfiziert worden? « der "Erfolg« der »Maßnahme « in Geld (»Gesamtersparnis: RM 88 439 800,00«) und Lebensmitteln ("Salz und Gewürze: $66393 \mathrm{~kg}$; Sago: $26552 \mathrm{~kg}$....) gemessen und die Ergebnisse bis 1951 hochgerechnet.

Ullrich und Bunke saßen in den Tötungsanstalten an einem erhöhten Tisch "genauso wie das Gericht hier «, erläuterte ein Zeuge. Sie musterten die nackt an ihnen vorbeiziehenden Deportierten unter dem Gesichtspunkt, wie ihr Tod ausgesehen hätte, hätten sie die nächste Stunde überleben dürfen, und notierten sich die erdachten Todesursachen, um diese anschließend sowohl in die bereits vorgefertigten "Trostbriefe « an die Hinterbliebenen als auch in die Sterbeurkunden der simulierten Standesämter einsetzen zu lassen. Nachdem die Türen des vermeintlichen Duschraums hinter den Opfern verriegelt waren, öffneten die Angeklagten Ullrich und Bunke die Gasflaschen.

\section{Der Prozeß}

Das Urteil im ersten Prozeß, der Freispruch also, ist zum Entsetzen der jetzigen Nebenkläger, die seinerzeit als solche nicht zugelassen worden waren - dank der Tätigkeit der "Absteckabteilungen « könne man nicht sicher sein, daß ihre Mütter tatsächlich von den angeklagten Ärzten, die die Benachrichtigungsbriefe unterschrieben hatten, ermordet worden waren -, I 967 von den Zuschauern mit Jubel aufgenommen worden.

Welche Erwartungen und Maßstäbe können heute, beinahe 20 Jahre später, an das wiederholte Verfahren gelegt werden, abgesehen davon, daß eine derartige Demütigung der Opfer und ihrer Kinder unterbleibt? Was wäre ein gerechtes Strafmaß für die jetzt 72 jährigen Ärzte, denen Beihilfe zum Mord in mindestens 4950 bzw. 1815 Fällen vorgeworfen wird? Welche Kriterien können angelegt werden, da es keine angemessene Strafe geben kann? Zumal nichts dafür spricht, eine Haftstrafe, wäre sie überhaupt vollstreckbar, werde auf die Angeklagten in irgendeiner Weise kathartisch wirken? Im Gegenteil: die Verstärkung ihrer Abwehr muß befürchtet werden. Leistet der Prozeß einen Beitrag, wieder Vertrauen in die Rechtsordnung zu setzen? Welche Rolle spielt in diesem Verfahren die Zeit? Der zeitliche Abstand zu den strafrechtlich zu würdigenden Taten von mittlerweile viereinhalb Jahrzehnten, zum ersten Prozeß von fast zwei Jahrzehnten? Welche Gefahren bergen möglicherweise die zeitgenössischen Positionen eines instrumentellen Geschichtsrevisionismus, nach denen derjenige in geschichtslosem Land die Zukunft gewinnt, wer die Erinnerung füllt, Begriffe prägt und die Vergangenheit deutet “ ${ }^{10}$ ? Inwiefern reproduziert das Verfahren über das unvermeidliche Maß hinaus Strukturen, die es zugleich inkriminiert?

In sozialwissenschaftlichen Untersuchungen von Kommunikationsstrukturen, die tiefenhermeneutisch verfahren, wird zumeist willkürlich ein kurzer Ausschnitt aus einem Kommunikationsprozeß herausgegriffen und so lange penibel analysiert und extensiv interpretiert, bis nichts zu erklären verbleibt, d.h. die Phantasien der Deutenden erschöpft sind, bzw. die äußeren Rahmenbedingungen der Interpretation Einhalt gebieten. Auf diese Weise erhält man prognostisch gehaltvolle Aussagen. In ähnlicher Weise war in den ersten zwei Verhandlungstagen sehr viel von dem 
ersichtlich, was in den folgenden Monaten nur ausgeführt und wiederholt werden

sollte.

Dem Gericht wurde Kampf angedient - über die Verhandlungsunfähigkeit des Beschuldigten Endruweit und über die drohende Verhandlungsunfähigkeit der übrigen Ärzte. Das Gericht hatte sich darauf vorbereitet. Ein Amtsarzt war von ihm in den Prozeß abgeordnet worden und jedem noch so häufig geäußerten Wunsch der Angeklagten nach einer Verhandlungspause wurde sofort, beflissen und großzügig stattgegeben. Mehr noch: die Vorsitzende Richterin, Johanna Dierks, nötigte den Angeklagten das Versprechen ab, ihr ja auch sofort zu sagen, wenn sie nicht mehr könnten. Und diese nickten dazu wie brave Kinder.

Angesichts dieser Niederlage eskalierte die Verteidigung ideologisch: Immanuel Kant, eine vergrößerte Prostata und 2I Kilogramm Übergewicht mußten dafür herhalten, einen Aussetzungsantrag zu begründen, das Gericht einer Verletzung des Art. 2 GG zu zeihen, da durch die Verhandlung - so soll der Philosoph gesagt haben - »die Wahrscheinlichkeit besteht, das Leben einzubüßen « (Verteidiger Wolfgang Meub). Johanna Dierks nahm es weise »zur Kenntnis «, Oberstaatsanwalt Hans Herrmann Eckert befand, »die Polemik liegt in der Natur der Sache«, und Johannes Riemann, Nebenklägervertreter, verwies in Zusammenhang mit $\$ 23$ ra StPO auf die Behandelbarkeit der Fettsucht. Nachdem der Antrag abgelehnt worden war, nahm der Verteidiger Meub das Recht in Anspruch, die Laienrichter der Befangenheit zu verdächtigen. Sie könnten möglicherweise Presseveröffentlichungen zum Prozeß rezipiert haben. Nachdem auch das geprüft und bestritten worden war, wurde anmaßend und submissiv zugleich für den Angeklagten Ullrich dessen "Kooperationsbereitschaft ", nämlich der Verzicht auf (offensichtlich unbegründete) Befangenheitsanträge, erklärt; an sie wurde allerdings sogleich die Bedingung geknüpft, »dieses Verfahren möglichst emotionslos durchziehen ..., es auf die knapp gehaltene Wiedergabe von Fakten beschränken zu wollen« und sich »persönlicher Wertungen « zu enthalten. Im übrigen sei in den vergangen fünfzehn Jahren der Verhandlungsunfähigkeit der Angeklagte Ullrich gezwungen gewesen, sein Leben aufs Spiel zu setzen, um seinen Beruf auszuüben.

Die stets latent explosive Mischung, die von einer Erziehung nach dem »Subordinationsprinzip (Ullrich) rührt, das übergangslose Oszillieren zwischen Trotz und Unterwürfigkeit, Großmannssucht und Strafangst, zwischen Servilität und den Keim des Scheiterns schon in sich bergenden und daher hilflosen aggressiven Ausbrüchen, die egozentrische Befangenheit, die für Gefühle der Scham keinen Platz läßt - dieses Verhalten der Angeklagten entschärfte Johanna Dierks so schnell, so nachhaltig, so geschickt, daß es einem oft den Atem verschlug. Dienten die Angeklagten mit all ihrer (vermeintlichen) Potenz dem Gericht einen der häufigen Kämpfe um Kinkerlitzchen an, gab sie ihnen lange Leine. Auf dieselbe Weise, in der eine kompetente Pädagogin in der Kindererziehung verfährt, installierte sie zwischen den Menschen auf der Anklagebank und den übrigen Beteiligten kontrafaktisch einen sozialen Konsens, unterstellte implizit ein geteiltes Interesse an der gemeinsamen Sache und stiftete sie so. Indem sie - gelegentlich gegen die begründeten Zweifel aller - den Angeklagten hinsichtlich ihrer Kooperationswilligkeit vertraute, weckte sie Vertrauen und erzielte eine nicht für möglich gehaltene Folgebereitschaft.

Da die anfängliche Nervosität und Spannung getilgt worden war durch Gelassenheit, Souveränität und Geduld, konnte in den folgenden Monaten, in denen die Aussagen von fast $5 \circ$ Zeugen, lebendigen wie verstorbenen, vernommen wurden, im Gerichtssaal das Böse in Ruhe banal werden. Diese Zeugen, ausnahmslos Tatbeteiligte, beleuchteten mit ihrer jeweiligen sozialen, kulturellen, beruflichen Prägung im 
Rücken mit Scheinwerferaugen das von der Arbeitsteilung gebildete Partikel ihrer Zuständigkeit in der Todesfabrikation. Im Schnittpunkt der Sichtweisen von Kraftfahrern, Maurern, »Brennern«, Standesbeamten, Chemikern, Krankenschwestern, psychiatrischen Gutachtern, Sekretärinnen, Juristen, ärztlichen- und Verwaltungsleitern der Anstalten entstand ein unerwartet homogenes Bild. Als bildeten die Zeugen noch heute die »verschworene Gemeinschaft", von der sie berichten, daß sie seinerzeit existierte, so als stünden sie nach wie vor miteinander in Verbindung und hätten sich untereinander abgesprochen, so als alterten sie nicht, spiele Zeit keine Rolle, traten sie vor Gericht auf; als handele es sich um ein einziges hartherziges Individuum, das noch heute erinnert, was es vor 47 Jahren gegessen hat, wer wem in welchem Restaurant ein Glas Wein spendierte, wer welche Erfindung zur Rationalisierung des "Betriebs « mit List gegen die Bürokratie durchsetzte, wie die Schüsse] beschaffen war, in der es sich wusch, wer mit wem eine Liebesaffäre unterhielt, welchen - unerhört gesitteten - Freizeitbeschäftigungen es nachging (Wäsche waschen, Gymnastik treiben und "gute« Bücher lesen), wie die vielen Betriebsfeiern und -ausflüge ausgestaltet waren, nicht jedoch an den Zweck seines Aufenthalts in der Tötungsanstalt, nicht an die, die als »Ballastexistenzen « »aus Liebe und Mitleid « (Ullrich) als Quelle von Zahngold nur von Wert waren. Dieser typische Zeuge konnte an seinem Arbeitsplatz nur 20 Zentimeter von dem Ort getrennt sein, in dem Tausende so zusammengepfercht erstickten, daß sie erst beim Öffnen der Türen umfallen konnten "wie Holz« (Zeuge Reuter) und nichts bemerken, denn "die Fenster gingen ja nach hinten raus« (Zeugin Dalades).

Spätestens in dieser Zeit setzten ein das Kettenrauchen in den Verhandlungspausen, das ungeheure Schlafbedürfnis in den Stunden nach dem Prozeß, der Wunsch, regelmäßig mit anderen über das Verfahren zu sprechen. Es war aber auch die Zeit, in der Journalisten während der Verhandlung Nachrichtenmagazine durchblätterten als säßen sie im Wartezimmer eines Frisörs, Schüler auf der Zuschauerbank kicherten als das System der Hauptstempel erläutert wurde, das erdacht worden war, um das Ausbeuten der Leichen zu vereinfachen.

\section{IV. "Juristengut *}

Die fatalen Urteile des Frankfurter Landgerichts und des BGH, in denen von "Geisteskranken ohne natürlichen Lebenswillen « und von »allerhöchstens einem Prozent niedergeführter Existenzen « die Rede ist, haben zusammen mit dem Heimtücke-Kriterium des $₫ 2$ I I StGB dazu beigetragen, daß sich das Gericht darauf eingelassen hat, erneut die Täuschungsbedürftigkeit und Täuschungsfähigkeit der Opfer der "Euthanasie « zum Gegenstand der Beweisaufnahme zu machen. So als rächte sich diese unmoralische Sichtweise, die die Logik der »Vernichtung lebensunwerten Lebens « in einem zentralen Punkt insofern reproduziert, als sie unterstellt, seelisch Leidende und hirnorganisch Kranke unterschieden sich prinzipiell von allen anderen Menschen, bildeten in Bezug auf ihre Ermordungsfähigkeit eine eigene, geringer zu achtende Klasse von Menschen, setzte diese Fortsetzung einer diskriminierenden Differenzierung unter den Opfern des nationalsozialistischen Massenmords der Phase prozessualer Ruhe ein Ende.

Als gäbe es in der BRD von nationalsozialistischer Ideologie unbeeinflußte Psychiater, die damals schon praktizierten, sondern Zahl, bestand das Gericht auf einem psychiatrischen Gutachter dieser Generation, der klären sollte, was unter einem »Endzustand«, einem "geistig Toten«, einer »niedergeführten Existenz und Schi- 
zophrenie u. a.m. zu verstehen sei, und wie viele derart Charakterisierter sich 1939/ 40 in den Heil- und Pflegeanstalten befunden hätten. Es kam wie es kommen mußte: Der Beauftragte und Professor emeritus, J. E. Meyer, Göttingen, verschwieg, daß er I 940 unter dem ersten psychiatrischen Ratgeber der KdF in Sachen »Euthanasie«, Max de Crinis, Berlin, promoviert hatte. ${ }^{11}$

Die antizipierte babylonische Sprachverwirrung kam zustande als Meyer - wie fast alle seiner Kollegen in solchen Dingen ungeschult - sich in den statistischen Maßzahlen zur Aufspaltung der Krankenhauspopulation verhedderte. Die Verteidigung erwachte aus ihrer Lethargie, übernahm begierig die diskreditierenden Begriffe einer ihrer gesellschaftlichen Rolle systematisch nicht bewußten Psychiatrie. Die mit einem hohen Aufwand an Suggestion vorgetragenen Behauptungen, das ermordete "Patientengut « habe aus "Ballastexistenzen«, aus »leeren, ausgebrannten Menschenhülsen ohne natürlichen Lebenswillen «, »unter dem Niveau von Tieren« bestanden und das sich anschließende Feilschen um Prozentangaben - die Rückzugsposition der Verteidigung - waren auf Dauer so schwer zu ertragen, daß man den Eindruck hatte, sie endeten niemals. Das Mittagessen der Verteidigung bestand an solchen Tagen aus Schnaps und ich hätte als Beobachterin der Sehnsucht nach Unmengen von Magenbitter eines Tages wohl auch nachgegeben, wäre da nicht ein Journalist gewesen, der eine kurze Schweigepause nutzte, um das Wort »Juristengut « in den Saal zu schleudern, und es mit diesem Einwortsatz fertig brachte, dem Spuk ein Ende zu bereiten. Die Solidarität der juristischen und psychiatrischen Laien im Gerichtssaal mit den Menschen, die unter die Kriterien der damaligen Meldebögen fallen, nötigte den Sachverständigen, sich bei den Klienten der Psychiatrie für seine niederführenden Etikettierungen zu entschuldigen, und zwang Verteidiger Meub, das gerade eroberte Terrain wieder preiszugeben. Seit den darauf folgenden Auseinandersetzungen um die Definitionsmacht der Psychiatrie, die es provoziert hatte, ist das Publikum nicht wieder in die passive Rolle der ersten Monate zurückgekehrt. Gleichwohl konnte nicht verhindert werden, daß andere psychiatrische Gutachten vorgetragen wurden, die ungestraft die »Endzustände im engen Sinn, im weiten Sinn und im anderen Sinn « ad nauseam durchdeklinierten.

Aus vielen NS-Verbrechensprozessen sind Strategien der Verteidigung bekannt, die zum Ziel haben, das Gericht mürbe zu machen. ${ }^{12}$ Wie in einer kriegerischen Materialschlacht regnete es auch hier fortan Befangenheits- und neue Beweisanträge. Der Gutachter wurde abgelehnt, weil er nicht reinrassisch arisch sei: er habe einen jüdischen Großvater; die Vorsitzende Richterin sei nicht neutral, weil sie die anschließenden Proteste des Publikums nicht gerügt habe; Oberstaatsanwalt Eckert sei befangen, weil er die Verbindungen zwischen der "Vernichtung lebensunwerten Lebens und der »Endlösung der Judenfrage« aufgezeigt habe. Den Einwand, die StPO sähe die Ablehnung des staatlichen Anklägers nicht vor, parierte Verteidiger Meub mit: Dann müsse sie eben geändert werden und dazu wolle er beitragen. Anträge wurden gestellt, einen Film in Augenschein zu nehmen: Johanna Dierks: "Was wollen Sie damit beweisen? « Wolfgang Meub: »Nichts«. Der Film müsse aber gezeigt werden, denn schließlich habe das Gericht dem Antrag des Nebenklägervertreters, einen Film vorzuspielen, auch stattgegeben.

I I In seıner Doktorarbeıt "Über organısche Hirnschäden und den Verfall der sıttlichen Haltung «, Archıv für Psychıatrıe, Bd. I 12, S. 368-384, wırd den dreı untersuchten Frauen eın "defektes Gemeınschaftsıch" beschemigt und dessen Sitz im Gehırn lokalisıert. Ihre Symptome, die zu dieser Diagnose führten: Sie gingen abends aus, engagierten sich politısch, waren mit ihrer beruflichen Unterprivilegierung unzufrieden und gaben auf Befragen zu, in Zeiten der Einsamkett sich selbst zu befriedigen

I2 Robert M. W. Kempner, in: Heiner Lıchtenstein, Im Namen des Volkes? Eine persönliche Bilanz der NS-Prozesse, Köln 1984, S. 8 
Die Chuzpe des Herrn Meub, die schon dem nicht-juristischen Prozeßbeobachter die Sprache verschlägt und ihn in einem merkwürdigen Rollentausch treibt, bei dem der Laie sich wider Erwarten in der Position dessen wiederfindet, der seine Hände schützend über die Rechtsordnung breitet, über die der Jurist meint, nach Maßgabe seiner Interessen disponieren zu können als sei er ein zweiter »Führer « - seine professionelle Schamlosigkeit irritiert erst recht eine Vorsitzende Richterin, die wie Johanna Dierks - den Eindruck vermittelt, auf ein besonderes Maß an professioneller Kompetenz großen Wert zu legen, mußte sie darauf doch ihre Berufskarriere gründen. Unprofessionalität beim Gegenüber scheint ihre einzige Achillesferse zu sein und so produziert sie Fehlleistungen, wenn Verteidiger Meub, als habe er's anno '68 bei Fritz Teufel gelernt, das Sprachspiel mit Hilfe von Tangentialisierungen verläßt, ${ }^{13}$ Ablehnungsanträge darauf stützt, ein Anonymus habe ihm gesagt, der Betreffende habe im vergangenen Jahr bei einem Vortrag an einer ganz bestimmten Stelle die Stimme gehoben oder eine Zeugin/ein Staatsanwalt habe am Telefon/auf der Straße zu einem Dritten etwas anderes gesagt als in der Hauptverhandlung etc., etc.

Man freut sich in diesen Situationen - wie so oft in diesem Prozeß - kein Richter zu sein; die - offenbar darin geübt - verpuppen, dauern die Tiraden länger, als Büsten ihrer selbst können sie stundenlang blicklos in den Saal gucken.

Rechtsanwalt Meub, dem sich seine Kollegen auf der Bank stets reflexhaft »anschließen «, ist ein angemessener Repräsentant seines Mandanten. Je länger der Prozeß währt, desto leichter ist Ullrich's Schweigen zu verschmerzen. Meubs Allmachtsphantasien, seine Larmoyanz und Schamlosigkeit, sein Zwang, „Widerworte $z u$ geben und seine wortreichen Umdeutungen der Täter zu den eigentlichen Opfern sind ein hinreichender Ersatz für »die Stimme seines Herrn".

Bei sprachlosen Schöffinnen und einem Oberstaatsanwalt, der sich sehr oft veranlaßt sieht, seine antifaschistische Gesinnung unter Beweis zu stellen, ist die Präsenz der Nebenkläger Simon und Strate und des Nebenklägervertreters Johannes Riemann ein Glück. Sie sind die einzigen in diesem Verfahren, die nachhaltig versucht haben, durch Filmaufnahmen, durch mühevolle Recherchen nach vernehmungsfähigen Verwandten, durch Zeugenbefragungen von Angehörigen, durch Familienbriefe und Proteste gegen die posthume Beleidigung ihrer Mütter in einem Prozeß ohne »betroffene Tatzeugen « (Oberstaatsanwalt Eckert) denjenigen, die als Markt- und Pfennigbeträge, als aggregierte "Desinfektion «, als sprachlich, wörtlich und soziale "Ab-Gestempelte", als aus dem Schornstein schlagende Flamme und als Rauch über der Stadt erscheinen, einen Platz in diesem Prozeß zu geben und sie endlich in unserer Vorstellung wiedererstehen zu lassen als das, was sie waren: Menschen wie Du und ich.

13 "Was meinen Sie dazu?* „Woher soll ıch das denn wissen!? « ... „Ihre Stellungnahme, bitte! «Tja, was sollen wir denn jetzt mal machen? « 